\title{
Biomarkers, interventions and healthy ageing
}

Almas Kenessary ${ }^{1}$, Zhaxybay Zhumadilov ${ }^{1}$, Talgat Nurgozhin ${ }^{1}$, David Kipling ${ }^{2}$, Mark Yeoman $^{3}$, Lynne Cox ${ }^{4}$, Elizabeth Ostler ${ }^{3}$ and Richard Faragher ${ }^{3 *}$

${ }^{1}$ Center for Life Sciences

Nazarbayev University

53 Kabanbay batyr ave

Astana, 010000

Republic of Kazakhstan

${ }^{2}$ Institute of Cancer and Genetics

School of Medicine

Cardiff University

Heath Park

Cardiff CF14 4XN

United Kingdom

${ }^{3}$ School of Pharmacy \& Biomolecular Science

Huxley Building

University of Brighton

Brighton

East Sussex BN2 4GJ

United Kingdom

${ }^{4}$ Department of Biochemistry

University of Oxford

South Parks Road

Oxford

OX1 3QU

United Kingdom

*Author for correspondence

\section{Contents}

Introduction

Why do we age?

One ageing process or many?

Crucial tests and interventions

Conclusions 


\begin{abstract}
Population ageing is probably the single most important healthcare challenge the developed and developing world will face in the $21^{\text {st }}$ Century. This is because the later part of the human life course is marked by the emergence of a wide spectrum of pathological impairments which increase morbidity and reduce quality of life. The processes driving these increases in mortality and morbidity are often conceptualised as highly complex and multicausal. Indeed, it has been suggested that there is no human "ageing process", only distinct, disease-specific mechanisms of pathology.

However, humans are not the only organisms within the biosphere to show ageing and the use of cross-species approaches has demonstrated that common ageing processes exist and allowed some of the common genetic pathways controlling them to be identified. Mutants in these pathways either delay or accelerate the development of late life diseases giving rise to extended healthy lives or progerias, respectively. These advances in fundamental understanding open opportunities for a more detailed investigation of the key causal mechanisms underlying ageing and the exploitation of that knowledge for improved interventions in later life.
\end{abstract}




\section{INTRODUCTION}

Over the past 150 years dramatic progress occurred in both public sanitation and our understanding of the causes of infectious disease. In combination, these discoveries produced the "epidemiological transition" - a very marked reduction in mortality in the early part of the life course due to a sharp fall in deaths from acute infectious disease, followed by a rise in both mortality and morbidity resulting from chronic degenerative conditions as a result of larger numbers of individuals surviving into later life [1].

The epidemiological transition is recognised as the single most important change to affect both the scale and patterning of human mortality to date. It has turned the problem of ageassociated diseases into the major one facing healthcare systems which were developed to deal primarily with purely exogenous causes of pathology (e.g. infectious disease or trauma). By way of example, it has been estimated that by 2050 , between $40-50 \%$ of the population of the western world and the Pacific Rim will be over the age of 60 [2]. Advancing chronological age is associated with an increased susceptibility to a wide range of degenerative conditions, which range in seriousness from the trivial to the fatal. All of these illnesses cost money and reduce the quality of life of those who suffer from them. The challenge posed by the ageing population in the $21^{\text {st }}$ century is thus to avoid both a sharp reduction in the quality of later life and steep increases in health spending.

The resource implications of dealing with the ageing population if conventional approaches are employed are very serious and dwarf the investment needed to improve health in early life. The public health measures that were responsible for the demographic transition were relatively cheap. For example, it has been estimated that the cost per life year saved by providing clean water in the USA was $\sim \$ 500$ (in 2003 dollars) [3]. In contrast, interventions such as stenting cost $\sim \$ 433,000$ per healthy life year gained (at 2007 values) [4]. In the same year (2007) the total cost of prescription medications for the British National Health Service was approximately $£ 8.4$ billion. Of the medicines purchased with this sum, patients over the age of 65 were prescribed $59.7 \%$ of all items dispensed by community pharmacists and $67.7 \%$ of items dispensed by doctors [5]. It has been estimated that $\sim 40 \%$ of the total budget of the British National Health Service is deployed combating the poor health consequences of ageing [6].

However a study from the RAND Corporation on future medical advances which might benefit the elderly showed that the potential exists for new treatments which are considerably cheaper. The best disease-specific example was a (hypothetical) drug which could reduce the incidence of Alzheimer's disease by one third. If such a drug existed then it could be used to provide one extra healthy year of life expectancy for $\$ 80,334$ (at 1999 values).In contrast, the RAND study estimated that a drug capable of adding ten years to healthy life expectancy could be used to purchase an extra healthy life year for $\$ 8,790$ (at 1999 values) [7]. This type of quantitative estimate of the potential benefits of understanding ageing and age-related disease highlights the need for both a better understanding of the fundamental biological basis of the process and the necessity of exploiting the knowledge gained so far in the production of better late life interventions.

\section{Why do we age?}

Ageing has been defined as a failure to maintain homeostasis under conditions of physiological stress [8]. This is accurate as far as it goes but the definition is insufficiently 
exclusive to provide a robust conceptual framework. Many researchers use that of Strehlar [9] who considered the defining features of ageing to be that it was universal, progressive, intrinsic and, above all, degenerative.

It also need to be stressed that even though the word 'ageing' is frequently applied to tissue components (as in 'cell ageing'), true ageing is something that can only happen to entire organisms. Formally speaking, it does not happen to proteins (which become adducted or damaged as a function of chronological dwell time in more or less damaging environments) and it does not happen to cells (unless the cell in question is something like yeast which is also an organism in its own right). Metazoan cells certainly undergo a range of changes over time and these changes almost certainly play causal roles in the ageing of the organism, but the cells themselves (whether mitotic or post mitotic) are not ageing in Strehlar's sense of the term. Rather, organismal ageing results from combinations of these intrinsic changes. What is the evolutionary rationale for ageing when it clearly compromises organismal survival?

Medawar (1952) [10] made what is still recognised as a fundamental insight into why ageing exists. He considered it to be a by product of the age-dependence of reproduction. This increases from zero (at the time of birth), peaks in young adult organisms (after puberty), and them decreases due to the increased likelihood of extrinsic mortality (e.g. predation, disease or accident). In natural conditions organisms rarely live to the age when ageing becomes apparent, thus their mortality is almost entirely dependent on extrinsic factors.

It follows that early-acting deleterious mutations are strongly selected against, since they affect the reproduction rate (or chances) of an organism. However, harmful mutations that occur later in the life course will not be exposed to such strong natural selection, as their carriers have already passed their genes to the next generation. A case in point would be Alzheimer's disease, which manifests itself in later life. Affected individuals are able to reproduce and, thus, potentially transfer the risk of developing pathology to their descendants.

The antagonistic pleiotropy theory of ageing [11] extended Medawar's initial observations on the age-dependence of reproduction by postulating the existence of pleiotropic genes i.e. alleles that have different effects on the survival of organisms during the life course. Such genes exert favourable effects on early life fecundity (when the effect of natural selection is strong), but deleterious ones in later life when the effect of natural selection is weak. The predictions of antagonistic pleiotropy (e.g. that fertility and longevity are negatively correlated) have been confirmed in experiments using the fruit fly Drosophila melanogaster. Postponing reproduction increased life expectancy and, conversely, decreased fertility was observed in long-lived flies [12]. Pleiotropic effects can occur both at the level of individual genes (e.g. p53 which plays a key role in the cellular anticancer mechanism at the cost of suppressing stem cell proliferation and survival) but also at the level of global processes such as cognition or tumour suppression.

The disposable soma theory, proposed by Kirkwood [13], has significant conceptual similarities to antagonistic pleiotropy. It postulates that longevity assurance mechanisms exist which are energetically expensive. Since energy is finite, organisms invest resources in somatic maintenance in line with their likely chances of survival. Since different species (sometimes even different individuals within species) have different external threat levels, this naturally leads to differential investment in somatic maintenance, differential reproductive rates and interspecies distinctions in the rate of ageing and maximum life span 
under protected conditions. For example, birds and bats live longer in comparison to land animals of similar size due to their ability to fly, which means that they experience less predator pressure.

Each of these theories has the potential to explain the variation in ageing rates seen in natural populations. However, not all the theories have resisted falsification to the same degree. Although there is some evidence that mutation accumulation plays a causal role in ageing this is quite limited and it appears unlikely that it is a major cause of ageing in Drosophila [14, 15]. The disposable soma theory has recently been subject to challenge with regard to the idea that the energy supply in the wild is limited. It has been suggested that the ability of organisms to dissipate heat, rather than the supply of energy per se, is the primary limit to reproductive success [16]. If this is accepted, then testable predictions arise that directly contrast with those posed by the disposable soma theory. In contrast, multiple lines of evidence are consistent with the idea of antagonistic pleiotropy as a broad-based ageing mechanism [17].

Antagonistic pleiotropy provides a useful starting framework for understanding the evolution of ageing, but an uncomplicated trade-off between early life fecundity and late life mortality should not be treated as the only factor determining lifespan in all species. For example, isolated populations of guppies (Poecilia reticulate) in the same river system in Trinidad live in either high or low predation environmental niches [18]. Classical antagonistic pleiotropy would predict that the fish populations in low predation environments should display relatively longer lifespans compared to those guppies in high predation niches. In fact, those guppies that evolved with predators mature earlier but also show longer lifespans than their counterparts in environments with low extrinsic mortality. This is difficult to reconcile with simple antagonistic pleiotropy and demonstrates that other factors (e.g. viability penalties for male sexual display) need to be factored into our standard evolutionary models for senescence [19].

Any theory which seeks to explain the existence of ageing must also be able to explain how it is possible for non-ageing organisms to evolve. Here again, antagonistic pleiotropy has provided an initial conceptual framework. In essence, an organism can be non-ageing if it becomes a quantitatively better replicator with time (in terms of offspring produced per unit of energy expended). This has the effect of reducing the overall potential benefits of mutations which increase early life fecundity but carry a late life fitness cost.

This fits well with a few examples of negligible senescence which exist in the natural world. A famous example of negligible senescence is the Cnidarian hydra, which is often considered to be immortal [20]. These organisms appear to be non ageing when growing in their asexual phase but undergo ageing after initiating transition to sexual reproduction. However asexual metazoans also undergo senescence and it would be wrong to consider ageing simply to be the "price paid for sex" [21].

The North Atlantic quahog (Arctica islandica) is perhaps the most extreme example of a complex organism that shows either negligible senescence or exceptional longevity. These molluscs have life spans in excess of 500 years [22]. The mechanisms by which the quahog achieves this exceptional lifespan remain unclear but, unlike many other ultra-long lived animals, the life cycle of Arctica (in particular the ability to readily harvest large populations of organisms of the same age from the same part of the sea bed) and its simple housing requirements render it a potentially tractable laboratory model [23]. 


\section{One ageing process or many?}

Evolutionary theory provides a useful conceptual framework for why ageing exists but is less helpful in explaining how the process operates. If any mutation which increases early life fecundity is selected for, even if this is associated with deleterious effects later on in the life course, then the number of processes which could contribute to ageing is potentially extremely large. Thus, in a complex organism, there could (in principle) be a distinct ageing mechanism for every tissue and every organ system. Classical epidemiologists have taken this view from time to time [24] and it resonates well with research programmes aimed at targeting specific diseases.

However, from the late 1980s onwards it became possible to isolate multiple mutants in common laboratory model organisms (eg nematode worms and fruit flies) which showed greatly extended lifespans [25-27]. Analysis suggested that these organisms were longer lived due to improved health status across multiple tissues and organ systems [28, 29]. Such observations are difficult to reconcile with a rigid "single pathology, single cause" model of later life dysfunction and are more consistent with a situation in which a few major "ageing" mechanisms drive the degeneration that characterises the latter period of the life course. If this is indeed the case, then it suggests that a realistic route to intervention in multiple late life pathologies is to target these ageing mechanisms rather than to focus efforts exclusively on cures for single age-associated diseases.

Many of the mutants that display significantly retarded ageing and extended lifespans compared to wild-type controls are found in the insulin/insulin-like growth factor signalling pathway (IIS mutants). These IIS mutations include the daf-2 mutant in C. elegans (a null mutation in the insulin/IGF1 receptor), chico (a Drosophila insulin receptor substrate protein mutant) and a variety of mutations in mice that affect either the insulin receptor in selected tissues, or global circulating insulin levels and insulin receptor substrates. However, the mechanisms by which these effects are produced were not initially clear. Reduced IGF-1 levels, increased cellular stress resistance, enhanced insulin sensitivity, altered body size, and improved anti-oxidant defences have all been proposed as contributing to the phenotype 28 , 29].

At least one potential mechanism, that ageing results from damage to the organism caused by oxidative stress (as postulated in the 'free radical' theory of ageing), now seems to be insecure [30].

If oxidative damage is indeed a major factor limiting lifespan, then animals with compromised antioxidant defences should show significant reductions in lifespan, and those with elevated protective or repair capacities should show extended lifespans. However, a large series of knockouts or knock-ins for genes involved in antioxidant protection have now been generated in both C.elegans and mice $[31,32]$. The general picture from these transgenic animals is that heterozygous C57BL/6 mouse knockouts of superoxide dismutase2 (Sod2), glutathione peroxidase-4 (Gpx4), thioredoxin-2 (Trx2) show increased levels of oxidative damage but no decrease in lifespan. Sod1 homozygous knockouts have shorter lifespans than wild-type littermates but overexpression of the enzyme fails to extend lifespan 
(a pattern replicated with $\mathrm{Sod} 2, \mathrm{Gpx} 4$, and catalase). As it stands, any simple assertion that oxidative damage alone forms the major constraint on lifespan seems unlikely to be valid.

Cross species genomic studies show that IIS mutants appear to down-regulate protein biosynthesis and enhance Phase I/Phase II xenobiotic metabolism (upregulation of cytochrome p450 genes, short-chain dehydrogenase/ reductases and glutathione-Stransferases) [33]. This has led to the idea that ageing results from macromolecular damage which has accumulated as a result of the toxic action of metabolic by-products [34]. Considered in these terms, lifespan is principally determined by the efficiency with which damaging species are removed or damaged macromolecules are repaired and recycled. The recent observation that mammalian lifespan can be extended using the mTOR inhibitor rapamycin is consistent with the idea that enhanced recycling of damaged cellular components is a prerequisite for extended organismal lifespan (since inhibition of TOR stimulates autophagy) $[35,36]$. This idea is also consistent with antagonistic pleiotropy because Phase I/Phase II metabolism is an energetically costly process to carry out and it is thus unlikely that selection has occurred for it to be more efficient than absolutely necessary.

It has been proposed that a major cause of the extended healthy lifespan of IIS mutant mammals is a reduced accumulation of senescence cells [37]. Senescence is the permanent entry of individual cells into a viable but non-dividing state, usually as the result of repeated cell division. The pathways which trigger this process are complex but the roles of the major molecular players are now relatively well understood [38]. Cell senescence can be observed in vitro in cells from a wide variety of different species and probably act as an anti-cancer mechanism [39]. Thus, senescence represents an example of antagonistic pleiotropy at the process level, limiting the growth of tumours in the early part of the organismal lifespan and contributing to organismal survival. However, cellular senescence is typically associated with the significantly upregulated secretion of proinflammatory factors (e.g. IL-6, IL-7, MCP-2, IL-8 and TNF-alpha) and matrix remodelling enzymes such as MMP-1 [40]. In vascular smooth muscle, the senescent cell phenotype is even more extreme and combines up-regulation of pro-inflammatory factors with the induction of an osteoblast-like phenotype,resulting in vascular calcification [41]. Cells with such radically altered phenotypes clearly have the potential to exert degenerative effects if their numbers reach a sufficiently high level in tissue.

The most rigourous test of the cell senescence hypothesis conducted to date involved deletion of senescent cells in vivo in the BubR1 progeroid mouse [42]. This resulted in delayed onset of a range of age-related pathologies, including cataracts and sarcopenia. The study has a number of limitations (most notably use of a non-standard genetic background) but provides proof of concept for more intensive investigation of replicative senescence in vivo.

\section{Crucial Tests and Interventions}

Exploiting novel model systems The ubiquitous nature of the ageing process has produced an enormous number of organisms which could potentially be studied and which might provide insights into how ageing operates. Of these, ultra-long-lived organisms have the potential to be the most informative, since their physiology may provide important clues as to how complex tissues can be organised which have either negligible senescence or survival time substantially in excess of those seen in humans. However, care needs to be taken in interpreting the results. 
For example, recent studies that found evidence that Arctica's extreme longevity is associated with resistance to oxidative damage. Protein carbonyl concentrations in A. islandica gill tissue are reduced compared with those of the shorter lived M. Mercenaria [43]. This may result from reduced ROS production in Arctica and may indicate a link between cellular redox homeostasis and life span. Alternatively, it may indicate nothing at all since another long-lived species (the naked mole rat) routinely has high, steady state levels of oxidative damage, levels of ROS production similar to its shorter lived relatives and exceptional antioxidant scavenging capacities [30]. In both species, more global studies of their cells' capacity for detoxification and ability to tolerate damage loads may be necessary if informative insights are to be gained. Arctica would prove a particularly useful species to study in this regard since it is closely related to molluscs with well characterised central nervous systems such as Lymnaea and Aplysia. Research on these species has informed much of our modern understanding of mammalian learning and memory.

Will elevated recycling prove to be a double-edged sword? Rapamycin produces a significant increase in lifespan, even if treatment is started in adulthood. Potentially, this provides proofof-principle that at least one drug (already licensed for clinical use) could slow the ageing process although how late in life such an intervention could be delivered remains a moot point. A central question thus becomes the extent to which this compound, or other related compounds (the so-called "rapalogues") can prevent or retard key aspects of morbidity. It is clear than rapamycin treatment has beneficial effects in transgenic mouse models of both Alzheimer and Huntington disease, probably via the induction of autophagy and the enhancement of proteostasis [44-46]. In themselves, these results are sufficient to justify sustained research into the therapeutic potential of compounds of this type. However, these results must be set against a series of studies which show that treatment of normal neurons with rapamycin inhibits some aspects of learning in a number of species. Thus in the normal situation, there is convincing evidence that rapamycin is detrimental to memory formation [47]. Reconciling these two areas of work requires detailed investigation the effects of mTOR inhibition (pharmacologically or otherwise) on deficits seen in normal brain ageing. This crucial work has yet to be carried out and is far from trivial even in simple model systems such as molluscs.

Drug development to tackle cell senescence. The evidence base that senescent cells do indeed contribute to organismal ageing appears to be solid. Thus a rational strategy would be to either develop drugs that remove such cells, or small molecules that block the worst of their pro-inflammatory effects. This would require a tractable animal model system where senescence can be efficiently and reproducibly induced in specific tissues of an otherwise normal animal. Such a system would be far more cost effective that attempting to work with populations of ageing animals, permit both an investigation of the effects senescent cells have on tissue structure and function, and would also provide a platform to assess the effect of drug-induced removal of senescent cells. It is to be hoped that such a system could be developed in the near future.

\section{Conclusions}

Ageing is often thought of as a highly complex multi-causal process and to a certain extent this is indeed the case. However, such thinking carries the unvoiced implication that the many potential mechanisms that could contribute to ageing at the different level of organisation of an organism (e.g. damage from endogenous oxygen radicals, protein glycation, epigenetic 
changes in DNA at the cellular level, neuroendocrine and immune system changes the whole organism level etc) all make roughly equal contributions to lifespan. The data emerging from the study of long-lived mutants animals does not seem to be consistent with such an interpretation but rather with one in which a small number of master regulators or key processes are responsible for major effects on lifespan. This improvement in our understanding of the fundamental biology of ageing is a very encouraging development which raises the prospect that we will be able to develop broad-spectrum treatment regimes for later life disease. At the current time, the most likely areas in which progress can be made rapidly would appear to be through study of the mTOR axis and the downstream effects of compounds which interdict it (e.g. rapamycin) and through targeted modulation of the phenotype of senescent cells. Progress in these areas will require much detailed analysis at both the genome and proteome levels and a long road to eventual drug development. However the fundamental biology tells us that this is a journey to a far-off destination and not a trip to never-never land. 


\section{REFERENCES}

[1] Wilmoth, J.R. (2000) Demography of longevity: past, present, and future trends. Experimental Gerontology 35, 1111-1129

[2] Lutz, W, Sanderson, W, Scherbov, S (1997). Doubling of world population unlikely. Nature 387, 803-5

[3] Cutler, D, Miller, G. (2005) The role of public health improvements in health advances: the twentieth-century United States. Demography 42, 1-22

[4] Venkitachalam, L. et al. (2011) Clinical and economic outcomes of liberal versus selective drugeluting stent use: insights from temporal analysis of the multicenter Evaluation of Drug Eluting Stents and Ischemic Events (EVENT) registry. Circulation 124, 1028-37

[5] NHS National Statistics (2008) Prescriptions Dispensed in the Community Statistics for 1997 to 2007: England. ISBN: 978-1-84636-223-1

[6] Summerfield, C. \& Babb, P. Eds. (2004) Social Trends 34 Published by London: TSO ISBN 011 6217324

[7] Goldman DP et al. (2005). Consequences of Health Trends and Medical Innovation For the Future Elderly. Health Affairs-Web exclusive available at http://content.healthaffairs.org.

[8] Comfort, A. (1979) The biology of senescence, Third Edition Published by Churchill-Livingstone.

[9] Strehlar, B (1977) Time, cells and aging. Second Edition Published by Academic Press

[10] Medawar, P.B. (1952) An Unresolved Problem in Biology Published by Lewis

[11] Williams, G.C. (1957). Pleiotropy, natural selection, and the evolution of senescence Evolution

11, 398-411

[12] Prowse, Partridge (1997) The effects of reproduction on longevity and fertility in male Drosophila melanogaster. Journal of Insect Physiology 43, 501-512

[13] Kirkwood, T. B. L. (1977) Evolution of Ageing Nature 270, 301-304

[14] Escobar JS, Jarne P, Charmantier A, David P (2008) Outbreeding alleviates senescence in hermaphroditic snails as expected from the mutation-accumulation theory. Curr Biol. 18:906-10

[15] Rose MR (2002) Evolution of late-life mortality in Drosophila melanogaster. Evolution 56:198291

[16] Speakman, JR. Król E. (2010) The heat dissipation limit theory and evolution of life histories in endotherms--time to dispose of the disposable soma theory? Integr Comp Biol. 50, 793-807

[17] Tabatabaie $V$ et al. (2011) Exceptional longevity is associated with decreased reproduction. Aging (Albany NY) 3:1202-5.

[18] Reznick D, Bryant M, Holmes D. (2006) The evolution of senescence and post-reproductive lifespan in guppies (Poecilia reticulata) PLOS Biol. 4:e7.)

[19] Godin, JG \& McDonough HE (2003) Predator preference for brightly coloured males in the guppy: a viability cost for a sexually selected trait. Behavioural ecology 14, 194-200

[20] Yoshida K et al. (2006) Degeneration after sexual differentiation in Hydra and its relevance to the evolution of ageing. Gene 385, 64-70

[21] Martínez DE, Levinton JS. (1992) Asexual metazoans undergo senescence. Proc Natl Acad Sci U S A. 89, 9920-3.

[22] Ridgway ID, Richardson CA, Austad SN. (2011) Maximum shell size, growth rate, and maturation age correlate with longevity in bivalve molluscs. J Gerontol A Biol Sci Med Sci. 66, 183-90

[23] Yeoman M, Scutt G, Faragher R. (2012) Insights into CNS ageing from animal models of senescence. Nat Rev Neurosci. 13, 435-45 
[24] Doll, R. (1997) There is no such thing as ageing British medical Journal 315, 1030-1032

[25] Klass, MR (1983) A method for the isolation of longevity mutants in the nematode Caenorhabditis elegans and initial results Mech Ageing Dev. 22, 279-86.

[26] Kenyon C et al. (1993) A C. elegans mutant that lives twice as long as wild type. Nature 366, 461-4.

[27] Friedman DB, Johnson TE (1988) A mutation in the age-1 gene in Caenorhabditis elegans lengthens life and reduces hermaphrodite fertility. Genetics 118, 75-86.

[28] Selman C et al. (2008) Evidence for lifespan extension and delayed age-related biomarkers in insulin receptor substrate 1 null mice. FASEB J. 22; 807-18.

[29] Gerstbrein et al. In vivo spectrofluorimetry reveals endogenous biomarkers that report healthspan and dietary restriction in Caenorhabditis elegans. Aging Cell 4; 127-37.

[30] Lewis KN, Andziak B, Yang T, Buffenstein R (2012) The Naked Mole-rat Response to Oxidative Stress: Just Deal With it. Antioxid Redox Signal. [Epub ahead of print]

[31] Pérez et al. (2009) Is the oxidative stress theory of aging dead? Biochim Biophys Acta. 1790; 1005-14.

[32] Gems D, Doonan R. (2009) Antioxidant defense and aging in C. elegans: is the oxidative damage theory of aging wrong? Cell Cycle 8, 1681-7.

[33] McElwee, J.J. et al. (2007) Evolutionary conservation of regulated longevity assurance mechanisms. Genome Biology 8, R132

[34] Gems, D. and McElwee, J.J. (2005) Broad spectrum detoxification: the major longevity assurance process regulated by insulin/IGF-1 signaling? Mechanisms of Ageing and Development 126, 381-387 [35] Wilkinson JE et al. (2012) Rapamycin slows aging in mice. Aging Cell 11; 675-82

[36] Wang SY et al. (2011) Core signaling pathways of survival/death in autophagy-related cancer networks. Int J Biochem Cell Biol. 43; 1263-6.

[37] de Magalhães JP, Faragher RG (2008) Cell divisions and mammalian aging: integrative biology insights from genes that regulate longevity. Bioessays 30, 567-78

[38] Itahana K, Campisi J, Dimri GP. (2004) Mechanisms of cellular senescence in human and mouse cells. Biogerontology 5,1-10

[39] Campisi J. (2011)Cellular senescence: putting the paradoxes in perspective. Curr Opin Genet Dev. 21, 107-12

[40] Coppé JP et al. (2010) The senescence-associated secretory phenotype: the dark side of tumor suppression. Annu Rev Pathol. 5, 99-118

[41] Burton DG et al (2009) Microarray analysis of senescent vascular smooth muscle cells: A link to atherosclerosis and vascular calcification. Exp Gerontol. 44, 659-65

[42] Baker DJ, (2011) Clearance of p16Ink4a-positive senescent cells delays ageing-associated disorders. Nature 479, 232-6.

[43] Ungvari Z et al. (2012) Resistance to Genotoxic Stresses in Arctica islandica, the Longest Living Noncolonial Animal: Is Extreme Longevity Associated With a Multistress Resistance Phenotype? J Gerontol A Biol Sci Med Sci. [Epub ahead of print]

[44] Ravikumar et al. (2004) Inhibition of mTOR induces autophagy and reduces toxicity of polyglutamine expansions in fly and mouse models of Huntington disease. Nat Genet 36, 585-95 [45] Spilman P (2010) Inhibition of mTOR by rapamycin abolishes cognitive deficits and reduces amyloid-beta levels in a mouse model of Alzheimer's disease. PLoS One 5, e9979. 
[46] Pierce A et al. (2012) Overexpression of heat shock factor 1 phenocopies the effect of chronic inhibition of TOR by rapamycin and is sufficient to ameliorate Alzheimer's-like deficits in mice modeling the disease. J Neurochem. [Epub ahead of print]

[47] Gafford GM, Parsons RG, Helmstetter FJ. (2011) Consolidation and reconsolidation of contextual fear memory requires mammalian target of rapamycin-dependent translation in the dorsal hippocampus. Neuroscience 182, 98-104 